\title{
Spatial analysis of SS population coverage based on emergency regional healthcare
}

\author{
Esra Morvan*, Bernadou Anne, Ludivine Gautier, Yassungo Silue and Dominique \\ Jeannel
}

Regional office, Sante publique France, Orléans, France

\section{Objective}

To analyse population coverage of syndromic surveillance(SS) based on emergency care data by studying i) the attractiveness of respectively SOS Médecins (Emergency care general practitioners) and Hospital emergency departments in the Centre-Val de Loire region and ii) the contribution of ecological deprivation factors in emergency access to healthcare.

\section{Introduction}

SOS Médecins France (SOS Med) is the first private and permanent network of general practitioners providing emergency care in France. Besides Hospital emergency departments (HED), SOS Med is therefore a major source of data for detecting and measuring nearreal-time health phenomena. The emergency services provided by the SOS Med have been subject to important changes in the recent years. Their services are enriched by a medical consultation center together with extended working hours. Besides, the south of the region is markedly affected by a declining number of medical practitioners This study was conducted to analyze the regional population coverage of emergency healthcare data provided by HED and SOS Med to the French syndromic surveillance system (SurSaUD ${ }^{\circledR}$ ) taking into account distance, health care offer, demographic factors and ecological deprivation factors.

\section{Methods}

An analysis of the activities and geographic attraction was carried out based on the data respectively provided by the three regional SOS Med and three HED (Bourges, Orléans and Tours). Quasi-Poisson regression modelling was used to identify the factors influencing the attractiveness of each organization. Next, the findings were refined through spatial analysis of the attractiveness of HED and SOS Med and analysis of the contribution of deprivation based on socioeconomical and healthcare facilities ecological indexes.

\section{Results}

In terms of age group, children under 2 years required the largest service consultations as well as seniors over 75 who sought more emergency visits at home. The SOS Med were almost always active in urban areas and at least once in two due to continuity of care. So they are an efficient source of general medical care given present work hours. Distance as an influential factor may explain the differences in attraction to the support type. The extent of the attraction appears in $36 \%$ SOS Med Bourges and $14 \%$ for SOS Med Orleans. Add the extent of attraction for SOS, remote consultation for SOS Med associations are a good use of care in general practice in present work hours scheme.

In terms of monitoring of epidemics, we note that the SOS Médecins associations are most active in winter, particularly during the seasonal epidemics of influenza. This can be explained by the fact of patient referrals during calls. The most serious cases are redirected to the ED and cases of general medicine to the SOS Médecins. It is also important to note that the attraction of ED of CHR Orléans covers more or less important a large part of the regional territory, which is not visible to the ED of $\mathrm{CH}$ Bourges. It should nevertheless be noted that the CHR Orleans a larger bed capacity than the CH Bourges.

\section{Conclusions}

This research has analysed the changes taking place in the SOS médecins associations in the Centre-Val de Loire region. Findings shows that these associations help ensure access to general medical care in a context of strongly reduced medical demography although with an uneven, primarily urban, geographical coverage. With better knowledge of the geographic span and sources and types of emergency care provision, further research can be undertaken to further refine and interpret the data.

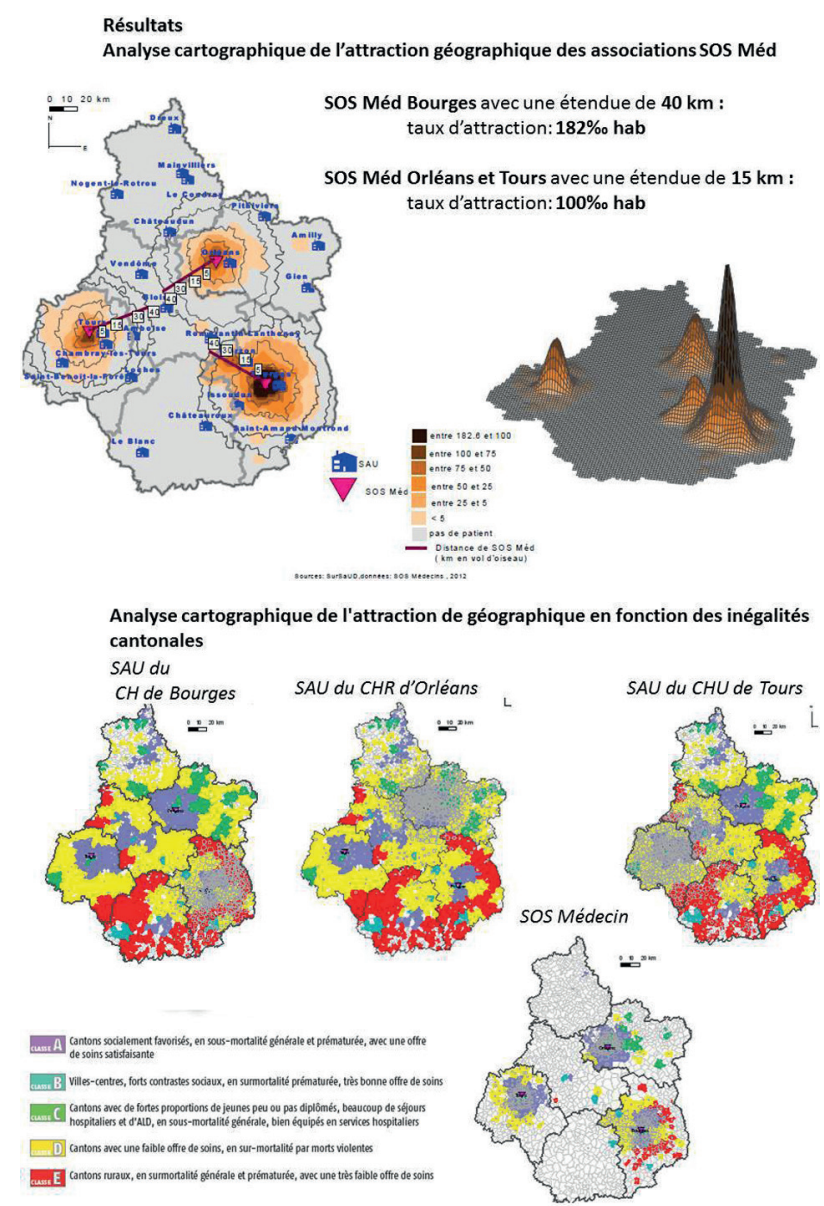

\section{Keywords}

emergency department; spatial analysis; ecological indexes; Centre-Val de loire; Sos Médecin (Emergency care general practitioners) 
ISDS 2016 Conference Abstracts

\section{Acknowledgments}

To SOS Médecins associations and all emergency department and ORS of the region Centre-Val de Loire for providing data

\section{References}

1.Rey G. Les tendances des inégalités de santé en France et en Europe Séminaire Sciences-Po "Réduire les inégalités de santé", 11 mai, Paris. 2011.

2.Bodenreider O, Lefebvre $\mathrm{O}$, Kohler F. Modèle pour l'étude de l'attraction exercée par un établissement hospitalier. Informatique et Santé. 1993;6.

3.Geniteau F, Guillouet C, Eloy A. Inégalités cantonales de santé en région Centre : une répartition territoriale des déterminants de santé : juillet 2010.

\section{*esra morvan}

E-mail: esra.morvan@ars.sante.fr 